\title{
PAPEL DE LA MEDICIÓN DE LA VELOCIDAD SISTÓLICA MÁXIMA EN ARTERIA CEREBRAL MEDIA EN LA ISOINMUNIZACIÓN RHESUS
}

\author{
Nayara López C. ${ }^{1}$, Roberto Rodríguez G. ${ }^{1}$, Antonio González G. ${ }^{1}$, Jesús Díez S. ${ }^{a}$ \\ 1 Departamento de Obstetricia y Ginecología, Hospital Universitario La Paz. Madrid, España.
}

a Estadístico, Departamento de Bioestadística, Hospital Universitario La Paz. Madrid, España.

\section{RESUMEN}

Objetivo: Evaluación del papel de la velocidad sistólica máxima en arteria cerebral media (VSM-ACM) en casos de isoinmunización Rh. Métodos: 67 casos de isoinmunización Rh, en el Hospital La Paz desde febrero del 2006 hasta agosto del 2009, con título de anticuerpos $\geq 1: 32$, afectación en embarazo previo y/o casos de isoinmunización anti- Kell, en los que se ha realizado medición de la VSM-ACM. Resultados: La capacidad de detección de anemia moderada-severa en base a la medición de VSM-ACM presenta: sensibilidad $80 \%$ (IC95\%: 59,8-100), especificidad y valor predictivo positivo $100 \%$, y valor predictivo negativo 85,7\% (IC95\%: 70,7-100). El coeficiente de correlación de Pearson entre la hemoglobina estimada y la real es de 0,71. Conclusión: La medición de VSM-ACM predice casos de anemia moderada y severa que son los clínicamente cruciales por la necesidad de actuación obstétrica activa en forma de transfusión intrauterina o finalización del embarazo.

\section{PALABRAS CLAVE: Anemia fetal, isoinmunización Rh, arteria cerebral media, transfusión intrauterina, cordocentesis}

\section{SUMMARY}

Objective: To evaluate the fetal middle cerebral artery peak systolic velocity (MCA-PSV) in the management of Rh isoimmunized pregnancies. Methods: 67 pregnancies complicated by Rh isoimmunization, in La Paz Hospital (Madrid) since 2006 February until 2009 August 2009, with maternal antibody titers $\geq 1: 32$, affected in previous pregnancies and/or anti-Kell isoimmunization, in which MCA-PSV has been measured. Results: For the detection of moderate-severe fetal anemia, Doppler ultrasonography of the middle cerebral artery had a sensitivity of $80 \%$ (Cl95\%: 59.8-100), a specificity and positive predictive value of $100 \%$, and a negative predictive value of $85.7 \%$ (CI95\%: 70.7-100). The Pearson correlation coefficient between estimated hemoglobin and real hemoglobin is 0.71 . Conclusion: The measurement of MCA-PSV predicts moderatesevere fetal anemia cases, which are the most important in the clinical management because of the need of active treatment with intrauterine transfusion or induction labor.

KEY WORDS: Fetal anemia, $\boldsymbol{R h}$ isoimmunization, middle cerebral artery, intrauterine transfusion, cordocentesis 


\section{INTRODUCCIÓN}

La etiología más frecuente de anemia fetal sigue siendo la aloinmunización de células rojas. Si bien tras la sistematización de la profilaxis con gammaglobulina anti-D disminuyó el número de casos, no se ha eliminado el problema. Ésta no es válida para el resto de antígenos irregulares y, debido a la inmigración, están reapareciendo en España casos de isoinmunización anti-D.

En embarazos complicados con isoinmunización $\mathrm{Rh}$, el feto puede desarrollar una anemia hemolítica que progresivamente desemboque en graves complicaciones sistémicas llegando al hidrops y muerte fetal (1). Si la anemia es diagnosticada y tratada precozmente con transfusiones intrauterinas, la tasa de supervivencia puede alcanzar el $90 \%(2)$.

La cordocentesis y la amniocentesis han sido los métodos empleados tradicionalmente en el seguimiento, pero ponen en riesgo también a fetos que no requerirán tratamiento intraútero. Hasta un $70-80 \%$ de los fetos sometidos a pruebas invasivas, sólo padecían anemia leve o carecían de anemia $(2,3)$.

Todo ello propició la búsqueda de marcadores ecográficos no invasivos. Los parámetros estáticos son consecuencia clínica de una anemia muy severa y se alejan del diagnóstico precoz buscado (4). Son los marcadores dinámicos la clave actual para la predicción de anemia fetal. La arteria cerebral media (ACM) es el vaso más idóneo para medir la velocidad de flujo tanto porque responde rápidamente a la hipoxemia, como por ser fácilmente visible con un ángulo de insonación cercano a $0^{\circ}$ entre el haz de ultrasonidos y la dirección del flujo sanguíneo.

Los parámetros de flujo se apoyan en una base fisiopatológica: el estado circulatorio hiperdinámico que conlleva la anemia. Así la capacidad de detección de anemia mediante medición de la velocidad sistólica máxima en arteria cerebral media (VSMACM) se limita a casos de anemia moderada y severa (2). Es a medida que la anemia se acentúa, cuando los mecanismos compensatorios actúan para mantener el equilibrio de oxígeno y nutrientes (4). Por otra parte, la relación entre VSM-ACM y hemoglobina fetal sigue una función cúbica por lo que la medición del pico sistólico es apropiada para casos de anemia moderada-severa, que son los que se corresponden con el tramo lineal de la curva (5). Son estos casos de anemia moderada y severa los clínicamente útiles al requerir una conducta activa.

El objetivo del estudio es evaluar el papel de la medición de la VSM-ACM en el manejo de fe- tos con riesgo de anemia debido a isoinmunización Rhesus.

\section{PACIENTES Y MÉTODO}

Estudio retrospectivo de los casos de isoinmunización Rh seguidos en el Hospital Universitario La Paz de Madrid, desde febrero de 2006 hasta agosto de 2009. Incluye 67 gestantes con 68 fetos con riesgo de anemia; 66 embarazos únicos con 1 gestación gemelar bicorial biamniótica.

Casos: Los casos recogidos presentan un título de anticuerpos $\geq 1: 32$, antecedente de afectación en gestación previa y/o son casos anti- Kell. Se excluyeron las gestaciones en las que se demostró la ausencia de posibilidad de afectación fetal por la prueba de Coombs directo. La edad gestacional de medición de VSM-ACM abarca desde la semana 19 de gestación hasta la 39, determinada por la historia menstrual confirmada por biometría fetal ecográfica.

Estudio Doppler: Las mediciones de VSM-ACM han sido realizadas obedeciendo las siguientes premisas $(2,3,5,6,7)$ : 1. Estudio del feto en periodo de descanso y ausencia de movimientos respiratorios. 2. Sección axial del cerebro, a nivel del esfenoides, paralelo y caudal al diámetro biparietal, en un corte que incluya tálamo y septum pellucidum. La arteria cerebral media se detecta por Doppler color, examinándola a 1-2 $\mathrm{mm}$ de su origen en la carótida interna y con zoom de más del $50 \%$ de la pantalla. 3. Ángulo de insonación lo más cercano posible a 0ㅜㅡ evitando corrección de ángulo. 4. Repetición de la medición al menos 3 veces, tomando la más alta puntuación como VSM-ACM (cm/s). 5. Equipo ecográfico apropiado para uso obstétrico (Voluson 730 Pro), con sonda abdominal de 4 a $8 \mathrm{MHz}$. Volumen de muestra en $2 \mathrm{~mm}$.

Interpretación de resultados: Se han seguido las curvas de normalidad publicadas por Mari y cols (2), para interpretar los valores medidos de VSMACM y predecir la anemia fetal que requiere una actitud obstétrica activa. Basado en las mismas, en la actualidad existe un programa en la página web de la Sociedad Española de Ginecología y Obstetricia que permite estimar el grado de anemia fetal a partir de los datos de VSM-ACM, número de transfusiones previas y edad gestacional (1). Según esta estimación, se han obtenido las cifras de hemoglobina calculada.

El test de referencia usado en el diagnóstico de anemia es la medida de las cifras de hemoglobina, bien de sangre fetal si se realiza cordocentesis previa a transfusión intrauterina, o bien las cifras neonatales de sangre de cordón o a las pocas horas 
del nacimiento si no se realiza cordocentesis. Así se obtienen los valores de hemoglobina real.

El grado de anemia fetal se ha determinado en base a la clasificación descrita por Mari y cols (2), en la que se considera anemia leve si la cifra de hemoglobina es $<0,84 \mathrm{MoM}$, moderada si $<0,65 \mathrm{MoM}$ y severa si $<0,55 \mathrm{MoM}$.

Los valores de hemoglobina y de VSM-ACM son expresados como múltiplos de la mediana (MoM) para ajustar el efecto de la edad gestacional en la medición.

En cuanto a la VSM-ACM, se ha considerado alta si la medición es $\geq 1,50$ MoM para edad gestacional y en límite alto de la normalidad si es $\geq 1,29$ MoM, correlacionándose respectivamente con anemia moderada-severa y con anemia leve. En fetos previamente transfundidos los puntos de corte empleados son diferentes: picos sistólicos $\geq 1,32 \mathrm{MoM}$ predicen anemia moderada-severa (8).

Análisis estadístico: Los datos se procesaron mediante SPSS versión 11.

Estudio descriptivo: para variables cuantitativas continuas se utilizó la media junto con la desviación estándar y para las cualitativas, frecuencias absolutas y relativas expresadas en porcentaje.

Estudio univariante: las comparaciones entre variables cuantitativas continuas se realizó mediante pruebas no paramétricas (Kruskal-Wallis o $\mathrm{U}$ de Mann-Whitney) y entre variables cualitativas mediante la prueba de $x^{2}$ o el test exacto de Fisher. La correlación entre variables cuantitativas continuas se estudió mediante el coeficiente de correlación de Pearson. El valor de $p<0,05$ indica la significación estadística.

\section{RESULTADOS}

De 10.000 partos anuales una media de $22 \pm 4$ embarazos se complican con isoinmunización $\mathrm{Rh}$, lo que supone una incidencia del 0,2 \%. El 75,8\% de los casos evaluados, engloba los tipos de isoinmunización anti-D, anti-E, anti-c y anti-K (Figura 1). El $10,3 \%$ de las gestantes en estudio era nuligesta. El 7,35\% tenía antecedente de transfusión previa en el marco de accidentes de tráfico, siendo sólo una nuligesta. El 35,3\% de las pacientes tenía historia de afectación en gestaciones anteriores y sólo el 5,9\% había recibido inmunoprofilaxis completa en embarazos previos.

Seguimiento del embarazo: Durante la gestación estudiada, el $24,2 \%$ presentó signos ecográficos de afectación fetal compatibles con anemia, llegando al hidrops en el 10,3\% de los casos (Figura 2). La semana de inicio de medición de la VSM-ACM presentó una media de $26,6 \pm 5,4$ semanas. La periodicidad de medición osciló entre 1 y 3 semanas en casos de puntuación alta y entre 2 y 5 semanas si los resultados se mantuvieron dentro de la normalidad.

De las 67 pacientes, a 8 se le administraron varios ciclos de inmunoglobulina hiperinmune $(11,7 \%)$ recibiendo 4 también tratamiento con plasmaféresis $(5,8 \%)$.

Un total de 15 fetos (22\%), requirieron transfusión intrauterina (TIU) (Figura 3). La semana media de realización de la primera TIU fue de $25,7 \pm 3,4$; de $27,6 \pm 3,8$ para la segunda, de $26 \pm 3,6$ para la tercera y de $26 \pm 2,8$ para la cuarta.

Un total de 22,9\% desarrolló alguna complicación secundaria a la TIU funicular: el 3,8\% hematoma de cordón, el $11,5 \%$ bradicardia mantenida que requirió cesárea urgente y el 7,6\% pérdida fetal en las 48 horas posteriores.

En cuanto a la última VSM-ACM evaluada durante la gestación, en el $63 \%$ de los casos se halló dentro de la normalidad y en el $22 \%$ fue alto para edad gestacional. La semana de gestación media a la que se realizó la última medición fue de 33,2 \pm

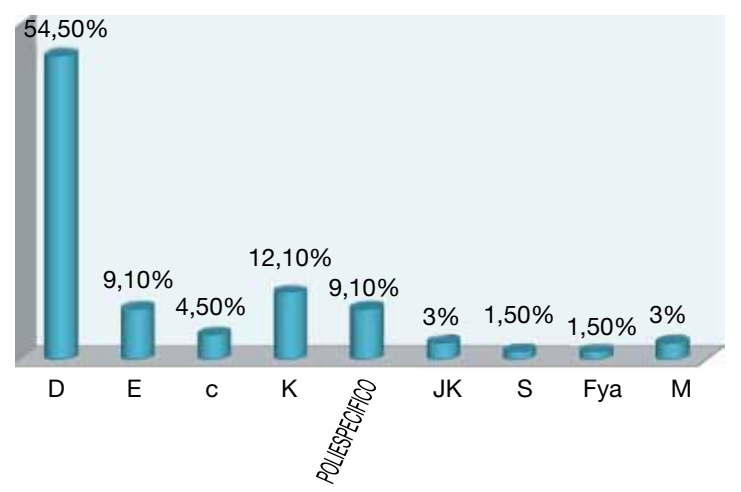

Figura 1. Tipos de isoinmunización (67 casos).

ASCITIS

HEPATOMEGALIA

ESPLENOMEGALIA

DERRAME PERICARDICO

GROSOR PLACENTARIO $>4 \mathrm{~cm}$

I. CARDIACA

HIDROTORAX

EDEMA PERICEFALICO

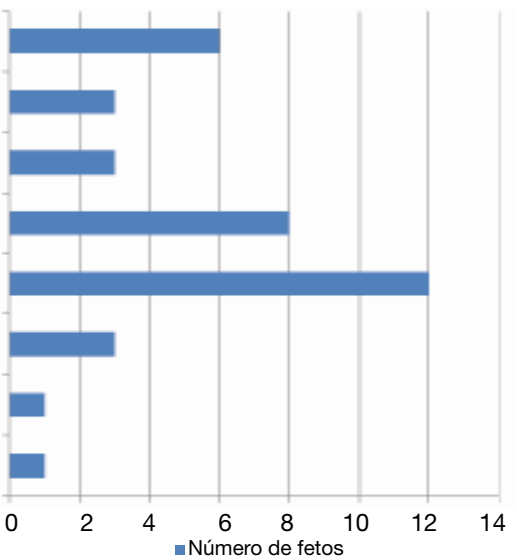

Figura 2. Signos ecográficos de afectación fetal por isoinmunización. 


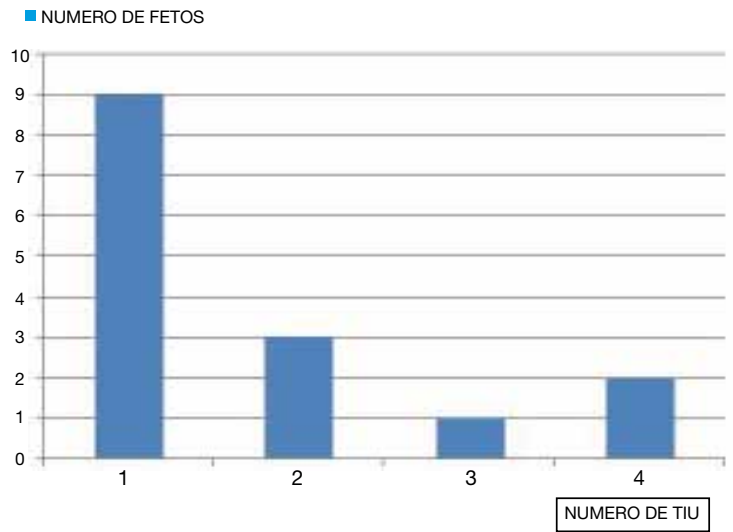

Figura 3. Número de transfusiones intrauterinas por feto.

3,9. En el $24,2 \%$ de los casos la medición se realizó por encima de la semana 35 y en el $31,8 \%$ con un intervalo de tiempo superior a 2 semanas entre la última medición y el parto.

La capacidad de detección de anemia moderada-severa de la medición de la VSM-ACM presenta, en base a los resultados obtenidos (Tabla I; Figura 4): sensibilidad del 80\% (IC95\%: 59,8-100); especificidad del $100 \%$; valor predictivo positivo del 100\%; valor predictivo negativo del $85,7 \%$ (IC95\%: 70,7-100).

Hubo tres falsos negativos. En todos se detectó anemia, pero sin correlación de grado exacta. La medición del pico sistólico estaba en el límite alto de la normalidad y predecía una anemia leve. En todas estas pacientes se llevó a cabo una conducta obstétrica activa basada en la VSM-ACM y otras consideraciones claves en isoinmunización. Todas fueron casos de isoinmunización anti-D. En una de ellas se realizó TIU por la existencia de un título de anticuerpos en ascenso, a pesar de varios ciclos semanales de inmunoglobulinas, unido al pico sistólico en límite alto de la normalidad. En los dos casos restantes, el antecedente de afectación en gestación previa, unido al hallazgo de signos ecográficos e hidrops, propició la indicación de finalizar la gestación tras la administración de corticoterapia a las 33 y 34 semanas respectivamente. Los dos neonatos desarrollaron anemización progresiva durante el ingreso y precisaron transfusión posterior.

Tomando la muestra de sangre obtenida por cordocentesis como referencia, la VSM-ACM detectó el $91 \%$ de los casos de anemia moderadasevera en la primera TIU; el $83,3 \%$ de los casos en la cordocentesis previa a la segunda transfusión, el $67 \%$ de los casos en la tercera y el $100 \%$ en la cuarta TIU. Se predijo anemia en todos los casos, pero la estimación falló en la correlación de grado. Los clasificó como anemia leve y la anemia real era moderada-severa.

Resultados neonatales: De 34 neonatos nacidos en el hospital, el 26,5\% presentó ictericia, el 2,9\% anemia y el $38,2 \%$ anemia con ictericia. El $17,6 \%$

\section{$\mathrm{Hb}$ Calculada}

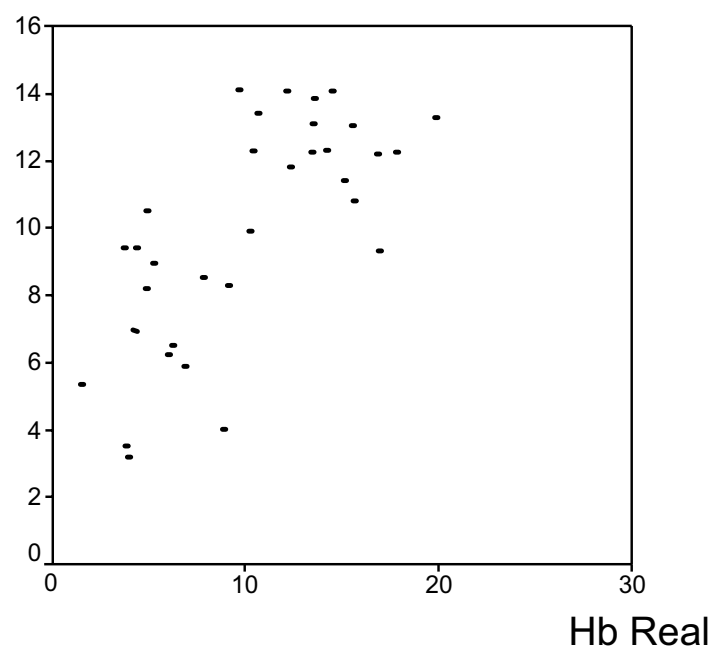

Figura 4. Correlación de la hemoglobina calculada en base a la medición de la VSM-ACM con la hemoglobina real, tras cordocentesis si se realiza transfusión intrauterina, o sangre de cordón si no se realiza. El coeficiente de correlación de Pearson es de 0,71 .

Tabla I

CORRELACIÓN DE GRADO ENTRE LA HEMOGLOBINA CALCULADA Y LA HEMOGLOBINA REAL

\begin{tabular}{llrr}
\hline & & \multicolumn{2}{c}{ Anemia real } \\
& & No o leve & Moderada o severa \\
\hline Anemia calculada & No o leve & $18(85,7 \%)$ & $3(14,3 \%)$ \\
& Moderada o severa & 0 & $(0 \%)$ \\
& & $12(100 \%)$ \\
\hline
\end{tabular}


murió intraútero, siendo la supervivencia global del $82,4 \%$. Se dispone de la evolución neonatal completa en 24 recién nacidos. De ellos, el $62,5 \%$ sufrió anemización progresiva y el 58,3\% requirió transfusión (Tabla II). El 45,8\% de los neonatos precisó fototerapia y/o gammaglobulina y el $54,2 \%$ restante exanguinotransfusión, con o sin fototerapia y/o gammaglobulina.

\section{DISCUSIÓN}

El incremento de casos de isoinmunización $R h$ junto con la instauración de la medición de VSMACM de forma sistemática propició el desarrollo del trabajo.

El periodo de tiempo estudiado incluye todos los casos con mediciones de la VSM-ACM realizadas por el servicio de fisiopatología fetal, desde las primeras mediciones en fetos enviados al final del embarazo, hasta la medición periódica en gestaciones seguidas desde el inicio.

El diseño ideal de un estudio para evaluar la correlación entre hemoglobina calculada según la medición de la VSM-ACM y hemoglobina real sería por cordocentesis, prueba considerada como patrón oro. Sin embargo, ello supondría someter a fetos sanos a los riesgos de una prueba invasiva siendo precisamente esto lo que se busca evitar al apostar por un test no invasivo. Ya existen trabajos con resultados al respecto por lo que este estudio se ha centrado en contrastar nuestros resultados con los disponibles en la bibliografía y evaluar la actuación obstétrica seguida $(2,6,9)$.

Se evalúan algunos fetos en más de una ocasión, si han sido sometidos a varias TIU y cuando se dispone también de datos de evolución perinatal. Para evitar esta limitación y respetar la asunción de independencia clave en el análisis estadístico, a la hora de correlacionar la hemoglobina calculada con la real, sólo se toman datos bien de la primera TIU si se realiza, o bien de sangre de cordón en los restantes casos. Así además se logra aumentar la potencia del estudio, al incluir tanto a fetos que requieren TIU sin datos neonatales, como a fetos que no necesitan TIU y de los que sí disponemos de datos al nacimiento.

La ventaja principal que aporta la medición de VSM-ACM es el seguimiento de fetos con riesgo de anemia de forma no invasiva. Sin embargo, es primordial emplearla solamente en estos casos de riesgo anémico, puesto que el pico sistólico es reflejo de una situación hemodinámica que, sacada de contexto, puede conducir a una actuación iatrogénica (5). De ahí que éste haya sido uno de los criterios de inclusión del estudio.

La finalidad fundamental de esta técnica es identificar aquellos fetos con anemia moderada-severa que requieren TIU y someter solamente a estos fetos a los riesgos de una técnica invasiva $(10,11,12)$.

La medición de la VSM-ACM se plantea como

Tabla II

\section{CORRELACIÓN DE LA ÚLTIMA MEDICIÓN DE LA VELOCIDAD SISTÓLICA MÁXIMA EN ARTERIA CEREBRAL MEDIA CON LA PRESENCIA O NO DE ANEMIA Y/O ICTERICIA, ANEMIZACIÓN DURANTE LA HOSPITALIZACIÓN Y NECESIDAD DE TRANSFUSIÓN NEONATAL}

\begin{tabular}{|c|c|c|c|c|c|c|c|}
\hline & & \multicolumn{2}{|c|}{$\begin{array}{l}\text { PATOLOGÍA } \\
\text { NEONATAL }\end{array}$} & \multicolumn{2}{|c|}{$\begin{array}{l}\text { ANEMIZACIÓN } \\
\text { EN INGRESO }\end{array}$} & \multicolumn{2}{|c|}{$\begin{array}{l}\text { TRANSFUSIÓN } \\
\text { NEONATAL }\end{array}$} \\
\hline & & NO & $\mathrm{SI}$ & NO & SI & NO & SI \\
\hline $\begin{array}{l}\text { Último } \\
\text { pico }\end{array}$ & Normal & $\begin{array}{l}10 \\
90,9 \%\end{array}$ & $\begin{array}{l}1 \\
9,1 \%\end{array}$ & $\begin{array}{c}6 \\
54,5 \%\end{array}$ & $\begin{array}{l}5 \\
45,5 \%\end{array}$ & $\begin{array}{c}7 \\
63,6 \%\end{array}$ & $\begin{array}{c}4 \\
36,4 \%\end{array}$ \\
\hline sistólico & Límite alto & $\begin{array}{c}2 \\
40 \%\end{array}$ & $\begin{array}{c}3 \\
60 \%\end{array}$ & $\begin{array}{l}3 \\
60 \%\end{array}$ & $\begin{array}{l}2 \\
40 \%\end{array}$ & $\begin{array}{l}3 \\
60 \%\end{array}$ & $\begin{array}{l}2 \\
40 \%\end{array}$ \\
\hline & Alto & $\begin{array}{c}1 \\
14,3 \%\end{array}$ & $\begin{array}{c}6 \\
85,7 \%\end{array}$ & $\begin{array}{l}0 \\
0 \%\end{array}$ & $\begin{array}{c}7 \\
100 \%\end{array}$ & $\begin{array}{l}0 \\
0 \%\end{array}$ & $\begin{array}{c}7 \\
100 \%\end{array}$ \\
\hline
\end{tabular}

El análisis $x^{2}$ demuestra una relación estadísticamente significativa entre las variables último pico sistólico y patología neonatal $(p<0,01)$, con asociación lineal entre ambas $(p<0,01)$. La relación entre la última medición de la VSM-ACM media y el desarrollo de anemización durante el ingreso es estadísticamente significativa en el análisis $x^{2}(p<0,05)$, con asociación lineal entre ambas $(p<0,05)$. Todos los neonatos que presentaron el ultimo pico sistólico alto requirieron transfusión durante su hospitalización, resultados estadísticamente significativos $(p<0,05)$, con una tendencia lineal significativa entre ambas $(p=0,01)$. 
una parte imprescindible del seguimiento de pacientes con isoinmunización $\mathrm{Rh}$, pero unido a la valoración de los antecedentes obstétricos, la determinación de anticuerpos y los signos ecográficos $(2,3,6,13,14,15)$.

Los resultados de nuestro estudio concuerdan con los datos publicados y confirman que existe una correlación inversa entre la hemoglobina fetal y VSM-ACM y que es una técnica válida para predecir anemia fetal moderada y severa junto con las consideraciones obstétricas descritas $(2,3,4,6,7)$. Todos los fetos sin anemia o con anemia leve presentaron una VSM-ACM $<1,50$ MoM. A pesar de incluir en el estudio mediciones de VSM-ACM por encima de la semana 35 de gestación, al correlacionar la hemoglobina calculada con la real, no se obtienen falsos positivos. Ello difiere de lo publicado en diversos estudios (16). También, a pesar de existir un porcentaje no despreciable de pacientes en las que el tiempo transcurrido desde la estimación de la hemoglobina hasta el nacimiento fue $\geq$ a 14 días, no se obtienen falsos positivos ni negativos (17). Si bien es cierto que todos son casos en los que no existe anemia o presentan sólo anemia leve, que es lo clínicamente importante, la correlación exacta de las cifras de hemoglobina es menos precisa en estas pacientes.

En el estudio se incluyen casos de isoinmunización anti-K, una gestación gemelar bicorial biamniótica y varios fetos a los que se somete a más de una TIU, corroborando así que la capacidad de la medición de la VSM-ACM para predecir anemia moderada-severa es aplicable a dichos casos, con las rectificaciones descritas en las TIU múltiples (8).

Entre los resultados conviene señalar que 2 de los 3 casos de falsos negativos presentan una anemia muy severa, en los que la sola presencia de hidrops es suficiente criterio de gravedad y reflejo de una situación hemodinámica severa y descompensada, que minimiza por innecesaria la poca precisión de la VSM-ACM en la determinación del grado de anemia.

\section{CONCLUSIÓN}

La medición de la VSM-ACM se plantea como un instrumento no invasivo clave en casos de anemia fetal moderada y severa que son los clínicamente cruciales por la necesidad de una actuación obstétrica activa.

\section{BIBLIOGRAFÍA}

1. Sociedad Española de Ginecología y Obstetricia. Pro- tocolo de isoinmunización eritrocitaria. 2008. Hallado en: www.sego.es.

2. Mari G, Deter RL, Carpenter RL, et al. Noninvasive diagnosis by Doppler ultrasonography of fetal anemia due to maternal red-cell alloimmunization. $\mathrm{N}$ Engl $\mathrm{J}$ Med 2000;342:9-14.

3. Scheier M, Hérnandez-Andrade E, Carmo A, Dezerega $\mathrm{V}$, Nicolaides $\mathrm{KH}$. Prediction of fetal anemia in rhesus disease by measurement of fetal middle cerebral artery peak systolic velocity. Ultrasound Obstet Gynecol 2004;23: 432-6.

4. Dukler D, Oepkes D, Seaward G, et al. Noninvasive tests to predict fetal anemia: a study comparing Doppler and ultrasound parameters. Am J Obstet Gynecol 2003;188:1310-4.

5. Mari G. Middle cerebral artery peak systolic velocity for the diagnosis of fetal anemia: the untold story. UItrasound Obstet Gynecol 2005;25:323-30.

6. Mari G, Adrignolo A, Abuhamad AZ, et al. Diagnosis of fetal anemia with Doppler ultrasound in the pregnancy complicated by maternal blood group immunization. Ultrasound Obstet Gynecol 1995;5:400-5.

7. Oepkes D, Seaward PG, Vandenbussche FP, et al. Doppler ultrasonography versus amniocentesis to predict fetal anemia. N Engl J Med 2006;355:156-64.

8. Detti L, Oz U, Guney I, et al. Doppler ultrasound velocimetry for timing the second intrauterine transfusion in fetuses with anemia from red cell alloimmunization. Am J Obstet Gynecol 2001;185:1048-51.

9. Alshimmiri MM, Hamoud MS, Al-Saleh EA, Mujaibel KY. Prediction of fetal anemia by middle cerebral artery peak systolic velocity in pregnancies complicated by rhesus isoimmunization. J Perinatol 2003;23:53640.

10. Bowman JM, Pollock JM. Transplacental fetal hemorrhage after amniocentesis. Obstet Gynecol 1985;66:749-54.

11. Holzgreve W, Tercanli S, Surbek D, Miny P. Invasive diagnostic methods. Fetal Medicine. London: Churchill Livingstone 1999:417-33.

12. Caine ME, Mueller-Heubach E. Kell sensitization in pregnancy. Am J Obstet Gynecol 1986;154:85-90

13. Nicolaides $\mathrm{KH}$, Bilardo $\mathrm{CM}$, Campbell S. Prediction of fetal anemia by measurement of the mean blood velocity in the fetal aorta. Am J Obstet Gynecol 1990;162:209-12.

14. Nicolaides $\mathrm{KH}$, Rodeck Ch, Mibashan RS, Kemp JR. Have Liley charts outlived their usefulness? Am J Obstet Gynecol 1986;155:90-94.

15. Nicolaides $\mathrm{KH}$, Soothill PW, Clewell WH, et al. Fetal haemoglobin measurement in the assessment of red cell isoimmunization. Lancet 1988;1:1073-5.

16. Zimmrmann R, Durig P, Carpenter RJ, Mari G. Longitudinal measurement of peak systolic velocity in the fetal middle cerebral artery for monitoring pregnancies complicated by red cell alloimmunisation: a prospective multicentre trial with intention-to-treat. $\mathrm{Br} \mathrm{J}$ Obstet Gynecol 2002;109:746-52.

17. Harrington K, Fayyad A. Prediction of fetal anaemia. Curr Opin Obstet Gynecol 2002;14:177-85. 